\title{
Mixed Path Ground Wave Propagation: 1. Short Distances
}

\author{
James R. Wait
}

\begin{abstract}
An expression is derived for the mutual impedance between two short vertical antennas on a flat earth with a straight boundary separating two media of differing electrical constants. After making some approximations that are valid at low and medium frequencies and where the antennas are not near the boundary, the integral formula for the field is evaluated for a wide range of the parameters. The numerical results computed in this paper are shown to bé in reasonably good agreement with experiment. Finally, the effect of the obliqueness of the boundary is considered by a refinement of the stationary phase evaluation of the integrals.
\end{abstract}

\section{Introduction}

Considerable interest has been shown recently in the propagation of ground waves over an inhomogeneous conducting earth. For many purposes, such as estimating coverage of broad cast transmitters, it is usually sufficient to assign an equivalent conductivity to the path. A simple and effective method for estimating this equivalent value from a conductivity profile of the path has recently been proposed by Suda [1]. ${ }^{1} \quad$ Another technique described by Kirke [2], which is similar, has been called the equivalent-distance method and is also simple to apply. The best known method, however, is due to Millington [3]. Although semiempirical it does appear to be valid for a wide range of frequencies and ground constants. It also predicts the recovery effect that occurs when the wave passes over a boundary from an area of poor conductivity to one of good conductivity. Millington does not provide a theoretical justification for his method, although he does indicate that his formulas for high frequencies are compatible with the expected behavior of the height-gain functions over the media on both sides of the boundary. Clemmow [4] in an elegant dissertation obtains a rigorous solution for a line source on flat earth parallel to a boundary separating two media. He makes a limited comparison of his formula with Millington's method, and shows that the agreement is good. In a more recent theoretical approach to the subject, Bremmer [5] formulates the problem in terms of an integral equation, which he solves by operational methods. After considerable manipulation he succeeds in showing that his result is mathematically equivalent to that of Clemmow. Bremmer considers several limiting cases and also establishes the validity of Millington's formulas at high frequencies.

It is the purpose of the present paper to extend Bremmer's result, with particular attention being paid to the phenomena at low and medium radiofrequencies where it does not seem possible to obtain convenient series expansions for the field by the Bremmer method. For the sake of completeness, the problem is reformulated in terms of mutual impedance between two antennas located on a flat earth, with a straight boundary separating two homogeneous media. In this case there is no doubt as to whether the reciprocity theorem is satisfied or not. The line joining the two antennas makes some angle with the boundary that should not be near zero. An integral equation is obtained for the field which is similar to one formulated by Feinberg [6] for propagation over a rough ground. In the present paper, the integral equation is solved by numerical means for a range of parameters that are appropriate for short distances at low and medium frequencies. It is intended to extend the calculations in a later paper to situations where the earth's curvature must be considered; that is, for distances in miles greater than about $50 \lambda^{1 / 3}$, where $\lambda$ is the wavelength in meters.

${ }^{1}$ Figures in brackets indicate the literature refêrences at the end of this paper. 


\section{Formulation}

The surface of the earth is considered to be flat and defined by $z=0$ in a simple cartesian coordinate system $(x, y, z)$. Points above the earth correspond to positive values of $z$. The earth medium to the left of a boundary line defined by $y=x \tan \theta$, as indicated in figure 1 , has a conductivity $\sigma$ and dielectric constant $\epsilon$. The earth medium for points to the right of the boundary line has a conductivity of $\sigma_{1}$ and dielectric constant $\epsilon_{1}$. Short hertzian dipole antennas are located at the points $\mathrm{A}$ and $\mathrm{B}$, which can be located anywhere along the $x$ axis just above the surface of the ground at $z=0$.

It is now assumed that a current $I_{a}$ applied at the terminals of antenna A would produce electric and magnetic fields $\bar{E}_{a}$ and $\bar{H}_{a}$ for the case when the earth media were homogeneous with electrical constants $\sigma$ and $\epsilon$. A time factor $\exp (i \omega t)$ is implied. The mutual impedance between antennas $\mathrm{A}$ and $\mathrm{B}$ for this homogeneous case is denoted as $Z_{a b}$. When the earth becomes inhomogeneous in the manner described above, the fields of antenna $\mathrm{A}$ with the same current $I_{a}$ become $\overline{E_{a}^{\prime}}$ and $\bar{H}_{a}^{\prime}$, and the mutual impedance becomes $Z_{a_{b}}^{\prime}$. It is convenient to regard the change from $Z_{a b}^{\prime}$ to $Z_{a b}$ as a consequence of the changes of the currents within the earth when it is transformed from a homogeneous to an inhomogeneous state. These currents will result in electric and magnetic fields $\bar{E}_{a}^{\prime}-\bar{E}_{a}$ and $\bar{H}_{a}^{\prime}-\bar{H}_{a}$ and a voltage $I_{a}\left(Z_{a b}-Z_{a b}^{\prime}\right)$ at the terminals of antenna B. A current $I_{b}$ is now considered to be applied at terminals of antenna B, and the resulting electric and magnetic fields are $\bar{E}_{b}$ and $\bar{H}_{b}$ over the homogeneous earth of constants $\sigma$ and $\epsilon$. It then follows from Ballentine's "corollary I" of the electromagnetic reciprocity theorem $[7,8]$ that

$$
Z_{a}^{\prime}-Z_{a b}=\frac{1}{I_{a} I_{b}} \iint_{S}\left[\bar{E}_{b} \times \bar{H}_{a}^{\prime}-\overline{E_{a}^{\prime}} \times \bar{H}_{b}\right]_{z} d x d y
$$

where the integration extends over the whole ground plane $S$, and the subscript $z$ indicates that the $z$ or normal component of vector products is taken.

A simplification is now made by introducing the concept of surface impedance [6,8], that is, the tangential electric and magnetic fields on the surface of the earth are assumed to be related by a complex constant of proportionality. More specifically,

$$
\left.\begin{array}{l}
E_{x} \simeq-{ }_{\eta} H_{y} \\
E_{y} \simeq{ }_{\eta} H_{x}
\end{array}\right\}
$$

where $\eta$, the surface impedance, is assumed to be equal to the value obtained for a plane wave at grazing incidence on the flat earth. It is given by

$$
\eta=120 \pi \frac{i \beta}{\alpha}\left[1+\frac{\beta^{2}}{\alpha^{2}}\right]^{1 / 2},
$$

where $\beta=2 \pi /$ wavelength, and $\alpha=\left(i \sigma \mu \omega-\epsilon \mu \omega^{2}\right)^{1 / 2}$, with $\mu=4 \pi \times 10^{-7}$. A subscript 1 is to be added to $\eta$ when the electric constants are $\sigma_{1}$ and $\epsilon_{1}$.

Equation (1) can now be written

$$
I_{a} I_{b} \Delta Z=\left(\eta-\eta_{1}\right) \iint_{S_{0}}\left(\bar{H}_{a t}^{\prime} \cdot \bar{H}_{b t}\right) d x d y
$$

where $\Delta Z=Z_{a b}^{\prime}-Z_{a b}$ is the change of the mutual impedance from the situation of the homogeneous earth of surface impedance $\eta$ to the inhomogeneous model indicated in figure 1 . The quantity $\bar{H}_{b t}$ is the tangential magnetic field of the antenna $\mathrm{B}$ over the homogeneous earth, and $\bar{H}_{a t}^{\prime}$ is the tangential magnetic field of the antenna $\mathrm{A}$ over the inhomogeneous earth. 


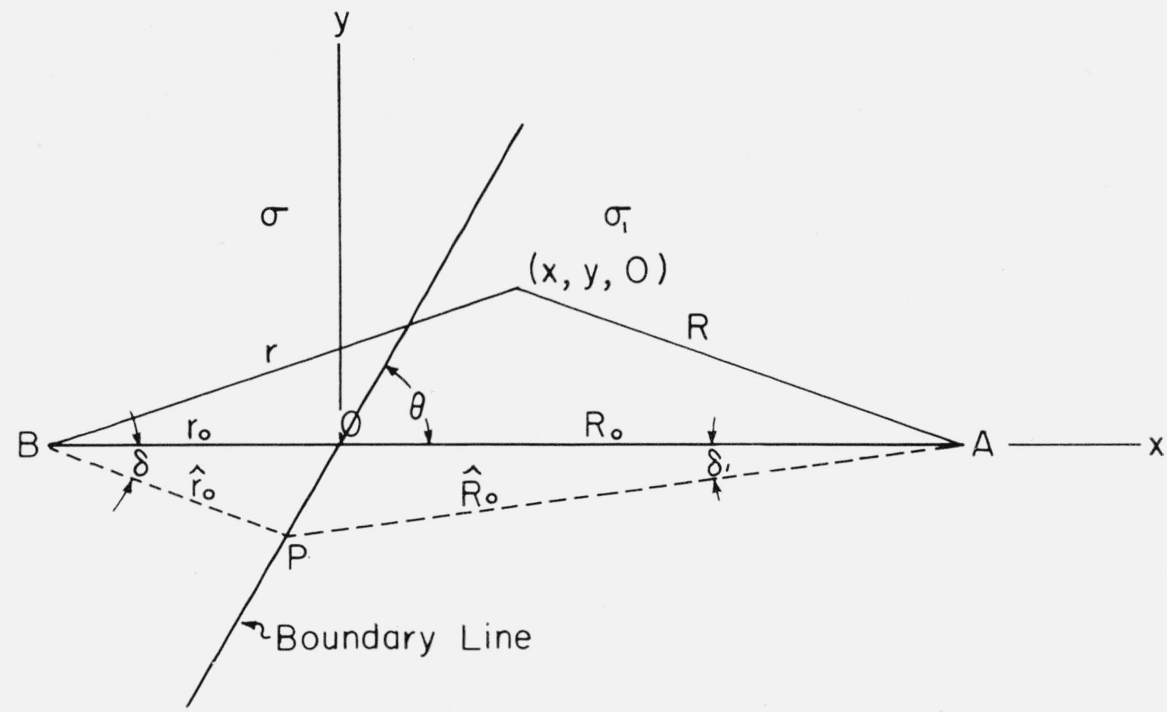

FIGURE 1. Schematic diagram of antennas $A$ and $B$ on a flat earth with a straight boundary separating two media with different conductivities.

The integration now extends over the surface $S_{0}$ to the right of the boundary line. Since $\bar{H}_{a t}^{\prime}$ is not actually known, eq (4) is actually a surface integral equation formulation of the problem. Before attempting to solve it, a simplification can be made if the principle of stationary phase is utilized.

Letting the hertzian antenna at $\mathrm{B}$ have an effective height $h_{b}$, the tangential magnetic field at $P$, a distance $r$ from $\mathrm{B}$, considering the earth as homogeneous and flat, is given by

$$
\bar{H}_{b t}=\frac{i \beta I_{b} h_{b}}{2 \pi r} e^{-i \beta r}\left(1+\frac{1}{i \beta r}\right) F(r, \eta)^{i}\left(\overline{i_{r}} \times \overline{i_{z}}\right),
$$

where $F(r, \eta)$ is Sommerfeld's ground-wave attenuation factor defined by

$$
F(r, \eta)=1-i 2 p^{1 / 2} e^{-p} \int_{\alpha=i p^{1 / 2}}^{\infty} e^{-\alpha^{2}} d \alpha,
$$

where

$$
p=+\frac{i \beta r}{2} \frac{\beta^{2}}{\alpha^{2}}\left(1+\frac{\beta^{2}}{\alpha^{2}}\right)=-\frac{i \beta r}{2}\left(\frac{\eta}{120 \pi}\right)^{2}
$$

is the numerical distance. Equation (6) is not exact, being an approximation valid for $\left.\beta^{2} \ll \alpha\right|^{2}$. $\quad \bar{i}_{r}$ and $\bar{i}_{z}$ are unit vectors in the directions of increasing $r$ and $z$, respectively. The function $F(r, \eta)$ has been treated numerically by Norton [9] and others. The tangential magnetic field $\bar{H}_{a t}^{\prime}$ at a distance $R$ from $A$ is of the form

$$
\bar{H}_{a t}^{\prime}=\frac{i \beta I_{a} h_{a}}{2 \pi R} e^{-i \beta R}\left(1+\frac{1}{i \beta R}\right) F^{\prime}\left(R, \eta, \eta_{1}\right)\left(\vec{i}_{R} \times \bar{i}_{z}\right),
$$

where $F^{\prime}\left(R, \eta, \eta_{1}\right)$ is some function of $R, \eta$, and $\eta_{1}$, and can be expected to be slowly varying compared to $e^{-i \beta R}$. $\quad \bar{i}_{R}$ is a unit vector in the direction of increasing $R$. 
Using eq (5) and (7) and denoting the angle between the vectors $\overline{i_{r}}$ and $\overline{i_{R}}$ as $\underline{\delta}$, it follows that the expression for the mutual increment $\Delta Z$ can be written

$$
\Delta Z=-\left(\eta-\eta_{1}\right) \frac{\beta^{2} h_{a} h_{b}}{4 \pi^{2}} \iint_{S_{0}} \frac{e^{-i \beta(r+R)}}{r R}\left(1+\frac{1}{i \beta r}\right)\left(1+\frac{1}{i \beta R}\right) F(r, \eta) \times F^{\prime}\left(r, \eta, \eta_{1}\right) \cos \delta d x d y,
$$

where

$$
\begin{aligned}
r & =\left[\left(r_{0}+x\right)^{2}+y^{2}\right]^{1 / 2} \text { and } \\
R & =\left[\left(R_{0}-x\right)^{2}+y^{2}\right]^{1 / 2} .
\end{aligned}
$$

The major contribution to the integrand occurs when the phase of the exponential term is nearly constant, since the others are relatively slowly varying. With this in mind, the exponent is expanded in a powers series in $y^{2}$ as follows:

and

$$
r+R \simeq r_{0}+R_{0}+\frac{y^{2}}{2}\left[\left(\frac{1}{r_{0}+x}\right)+\left(\frac{1}{R_{0}-x}\right)\right] \text { for } R_{0}>x>0
$$

$$
r+R \simeq r_{0}-R_{0}+2 x+\frac{y^{2}}{2}\left[\left(\frac{1}{r_{0}+x}\right)+\left(\frac{1}{x-R_{0}}\right)\right] \text { for } x>R_{0}
$$

where terms in $y^{4}, y^{6}$, etc., are neglected. A further approximation to the integrand in eq (8) is to replace $(1+1 / i \beta r)$ and $(1+1 / i \beta R)$ by unity. This will be justified if the antenna $\mathrm{A}$ or $\mathrm{B}$ is not near the boundary (i. e., $\beta R_{0}$ and $\beta r_{0} \gg 1$ ). This is essentially equivalent to stating that the effect of the induction field of the antennas is not considered so far as the boundary is concerned.

The integral expression for $\Delta Z$ now has the following approximate form:

$$
\Delta Z=+\frac{\left(\eta_{1}-\eta\right) \beta^{2} h_{a} h_{b}}{(2 \pi)^{2}} \frac{e^{-i \beta\left(r_{0}+R_{0}\right)}}{\left(r_{0}+R_{0}\right)^{\frac{1}{2}}} \int_{x=0}^{R_{0}} \frac{F\left(r_{0}+x, \eta\right) F^{\prime}\left(R_{0}-x, \eta, \eta_{1}\right)}{\left(r_{0}+x\right)\left(R_{0}-x\right)} \times\left[\int_{-\infty}^{+\infty} e^{-i \alpha^{2} y^{2}} d y\right] d x
$$

where

$$
\alpha^{2}=\frac{\beta}{2}\left[\frac{R_{0}+r_{0}}{\left(r_{0}+x\right)\left(R_{0}-x\right)}\right] .
$$

The integration over $x$ only extends from 0 to $R_{0}$, because the contribution from the integrand for $x>R_{0}$ is negligible due to the presence of the rapidly varying function $e^{-2 i \beta x}$.

\section{Numerical Solution}

The mutual impedance $Z$ between the two short antennas $A$ and $B$ for the homogeneous ground of electrical constants $\sigma$ and $\epsilon$ is given by

$$
Z=\frac{h_{a} h_{b} i \mu \omega}{2 \pi\left(r_{0}+R_{0}\right)} e^{-i \beta\left(r_{0}+R_{0}\right)} F\left(r_{0}+R_{0}, \eta\right)\left[1+\frac{1}{i \beta\left(r_{0}+R_{0}\right)}-\frac{1}{\beta^{2}\left(r_{0}+R_{0}\right)^{2}}\right]
$$

It is now convenient to express the mutual impedance $Z^{\prime}$ between the antennas on the inhomogeneous ground as follows:

$$
Z^{\prime}=\frac{h_{a} h_{b} i \mu \omega}{2 \pi\left(r_{0}+R_{0}\right)} e^{-i \beta\left(r_{0}+R_{0}\right)} F^{\prime}\left(r_{0}+R_{0}, \eta_{1}, \eta\right)\left[1+\frac{1}{i \beta\left(r_{0}+R_{0}\right)}-\frac{1}{\beta^{2}\left(r_{0}+R_{0}\right)^{2}}\right]
$$


where $F^{\prime}$ is the unknown attenuation function. The latter bracketed term can be replaced by unity because $\beta\left(r_{0}+R_{0}\right)$ is large compared to 1 . Employing eq (10), (11) and (12), it follows that

$$
F^{\prime}\left(r+R_{0}, \eta, \eta_{1}\right) \simeq F\left(r_{0}+R_{0}, \eta\right)-i \epsilon_{0} \omega\left(\eta-\eta_{1}\right)\left(\frac{d^{1 / 2}}{2 \pi i \beta}\right) \int_{0}^{R_{0}} \frac{F\left(r_{0}+x, \eta\right) F^{\prime}\left(R_{0}-x, \eta_{1}, \eta\right) d x}{\left[\left(r_{0}+x\right)\left(R_{0}-x\right)\right]^{\frac{1}{2}}}
$$

This is an integral equation for $F^{\prime}$. It is immediately apparent when $x<0$ such that $\mathrm{A}$ and $\mathrm{B}$ are to the left of the boundary

$$
F^{\prime}\left(r_{0}+R_{0}, \eta, \eta_{1}\right) \simeq F\left(r_{0}+R_{0}, \eta\right) .
$$

In other words, subject to our stationary phase approximation, the attenuation function $F^{\prime}$ between $\mathrm{A}$ and $\mathrm{B}$, when they are located on one side of the boundary, is characteristic only of the electrical constants $\sigma$ and $\epsilon$ of the homogeneous ground between them. For exactly the same reason, the function $F^{\prime}\left(r_{0}+x, \eta, \eta_{1}\right)$, where it occurs in the integrand of equation, for the general case of $x>0$, can be replaced by $F\left(r_{0}+x, \eta_{1}\right)$, which is characteristic of propagation from $\mathrm{A}$ to the point $x(>0)$ over a homogeneous ground of electrical constants $\sigma_{1}$ and $\boldsymbol{\epsilon}_{1}$.

After a change of variable, the final expression for $F^{\prime}$ can be written in dimensionless form, yielding

with ${ }^{2}$

$$
F^{\prime}=F\left(p_{0}\right)-i\left(\frac{p_{0}}{\pi}\right)^{1 / 2}(1-\sqrt{K}) \int_{0}^{p_{0} V / K} \frac{F(p) F\left(p_{0}-K p\right)}{\sqrt{p\left(p_{0}-K p\right)}} d p
$$

and

$$
F(p)=1-i 2 p^{1 / 2} e^{-p} \int_{i p^{1 / 2}}^{\infty} e^{-\alpha^{2}} d \alpha
$$

$$
\begin{gathered}
p_{0}=-\frac{i \beta\left(r_{0}+R_{0}\right)}{2}\left(\frac{\eta}{120 \pi}\right)^{2} \\
K=\frac{\sigma_{1}+i \omega \epsilon_{1}}{\sigma+i \omega \epsilon} \text { and } V=\frac{R_{0}}{R_{0}+r_{0}} .
\end{gathered}
$$

The above expression for $F^{\prime}$ is then given in terms of the attenuation function $F\left(p_{0}\right)$, characteristic of propagation between $\mathrm{A}$ and $\mathrm{B}$ on a homogeneous ground, and a correction term that accounts for the change of the electrical constants of the ground to the right of the boundary. It should be noted that $p_{0}$ is the "numerical distance" between A and B with regard to the earth medium to the left of the boundary, whereas $p_{0} V / K$ is the numerical distance between the boundary to the point $B$ with regard to the earth medium to the right of the boundary. Unfortunately this expression, which involves an integration over products of error functions, is not readily expressible in closed form. However, the integral can be approximated in certain limiting cases. For example, when $p_{0}(1-V)$ and $p_{0} V / K$ are small compared to 1 , the power series expansion for the functions $F(p)$ and $F\left(p_{0}-K p\right)$ can be employed. This has the form

$$
F(p)=1-i(\pi p)^{1 / 2}-2 p+i \sqrt{\pi} p^{3 / 2} \pm \cdots \cdots
$$

It then follows without difficulty that

$$
F^{\prime}=F\left(p_{0}\right)+2 i\left(\frac{p_{0}}{\pi}\right)^{1 / 2}\left(1-\frac{1}{\sqrt{K}}\right) \tan ^{-1}\left(\frac{V}{1-V}\right)-2 p_{0}(1-\sqrt{K})\left[\frac{1}{K}(1-\sqrt{1-V})+\left(\frac{V}{K}\right)^{1 / 2}\right],
$$

\footnotetext{
${ }^{2}$ For large distances, the function $F$ must be modified to account for earth curvature. This will be considered in section 2 .
} 
plus terms in $p_{0}^{3 / 2}, p_{0}^{2}$, etc. Another special case is when $p_{0}$ is large compared to 1 , so that only the leading term in the asymptotic expansion of $F\left(p_{0}-K p\right)$ need be considered; and furthermore, $V$ is assumed to be small compared to unity. In this instance

$$
\frac{F\left(p_{0}-K p\right)}{\left(p_{0}-K p\right)^{1 / 2}} \simeq-\frac{1}{2\left(p_{0}-K p\right)^{3 / 2}} \simeq-\frac{1}{2 p_{0}^{3 / 2}}
$$

The power series formula for $F(p)$ is then employed, yielding, after integrating term by term:

$$
\frac{F^{\prime \prime}}{F\left(p_{0}\right)} \simeq 1-\frac{2 i(1-\sqrt{K})}{\sqrt{\pi}}\left[p_{1}^{1 / 2}-\frac{i \sqrt{\pi} p_{1}}{2}-\frac{2 p_{1}^{3 / 2}}{3}+\frac{i \sqrt{\pi}}{4} p_{1}^{2}+\frac{4}{3} \frac{p_{1}^{5 / 2}}{5}-\frac{i \sqrt{\pi}}{2} \frac{p_{1}^{3}}{6}-\frac{8}{15} \frac{p_{1}^{7 / 2}}{7}+\cdots\right],
$$

where $p_{1}=p_{0} V / K$.

There are probably other limiting cases which will enable the integration with respect to $p$ to be effected; however, it is believed that for application to low and medium radiofrequencies the values of $p_{0}$ are in general neither large nor small compared to one. With this in mind, it is considered desirable to evaluate $F^{\prime}$ by a numerical integration for a range of $p_{0}, V$, and $K$. It should be noted that, for the general case, $p_{0}$ and $K$ are complex so there are actually five parameters to consider for the two-media problem. In this paper, attention will be restricted to frequencies where the displacement currents in the ground can be neglected. That is, the ratios $\epsilon \omega / \sigma$ and $\epsilon_{1} \omega / \sigma_{1}$ are assumed to be small compared to unity, therefore $p_{0}$ $=\pi\left[\left(R_{0}+r_{0}\right) / \lambda\right]\left(\epsilon_{0} \omega / \sigma\right)$ and $K\left(=\sigma_{1} / \sigma\right)$ are real quantities. This is usually justified for frequencies less than $1,000 \mathrm{kc}$ for typical ground constants [10].

Employing the numerical values of the function $F(p)$ and $F\left(p_{0}-K p\right)$. the integral in eq (14) is evaluated by a graphical method. The function $F^{\prime}\left(p_{0}, V, K\right)$ is then plotted as a function of $p_{0}$ from 0.1 to 5 for various values of $K$ and $V$ in figures 2 to 11 . It is believed that the results plotted in this form can be adapted to a large number of practical situations. The curves are not shown beyond $p_{0}=5$, since this usually corresponds to higher frequencies where displacement currents are nonnegligible.

It is of considerable interest at this stage to compare these numerical results with those computed using Millington's method [3]. His empirical formula for $F^{\prime}\left(p_{0}, V, K\right)$ in the notation of the present paper would read

$$
F^{\prime}\left(p_{0}, V, K\right)=\left[F\left(p_{0} / K\right) F\left(p_{0}\right) \frac{F\left[p_{0}(1-V)\right] F\left(p_{0} V / K\right)}{F\left[p_{0}(1-V) / K\right] F\left(p_{0} V\right)}\right]^{1 / 2}
$$

Values computed from this formula for the cases $K=2$ and $K=\infty$ are indicated by $\bigcirc$ and - respectively, on figures 2 to 11 . The agreement is quite reasonable, and therefore further support is given to the validity of the Millington method, which has been known to predict correctly, within $1 \mathrm{db}$ or so, the attenuation over certain mixed paths. It is believed, however, that differences are not of sufficiently small order to be neglected if fairly precise values of the fields in amplitude and phase are required. 


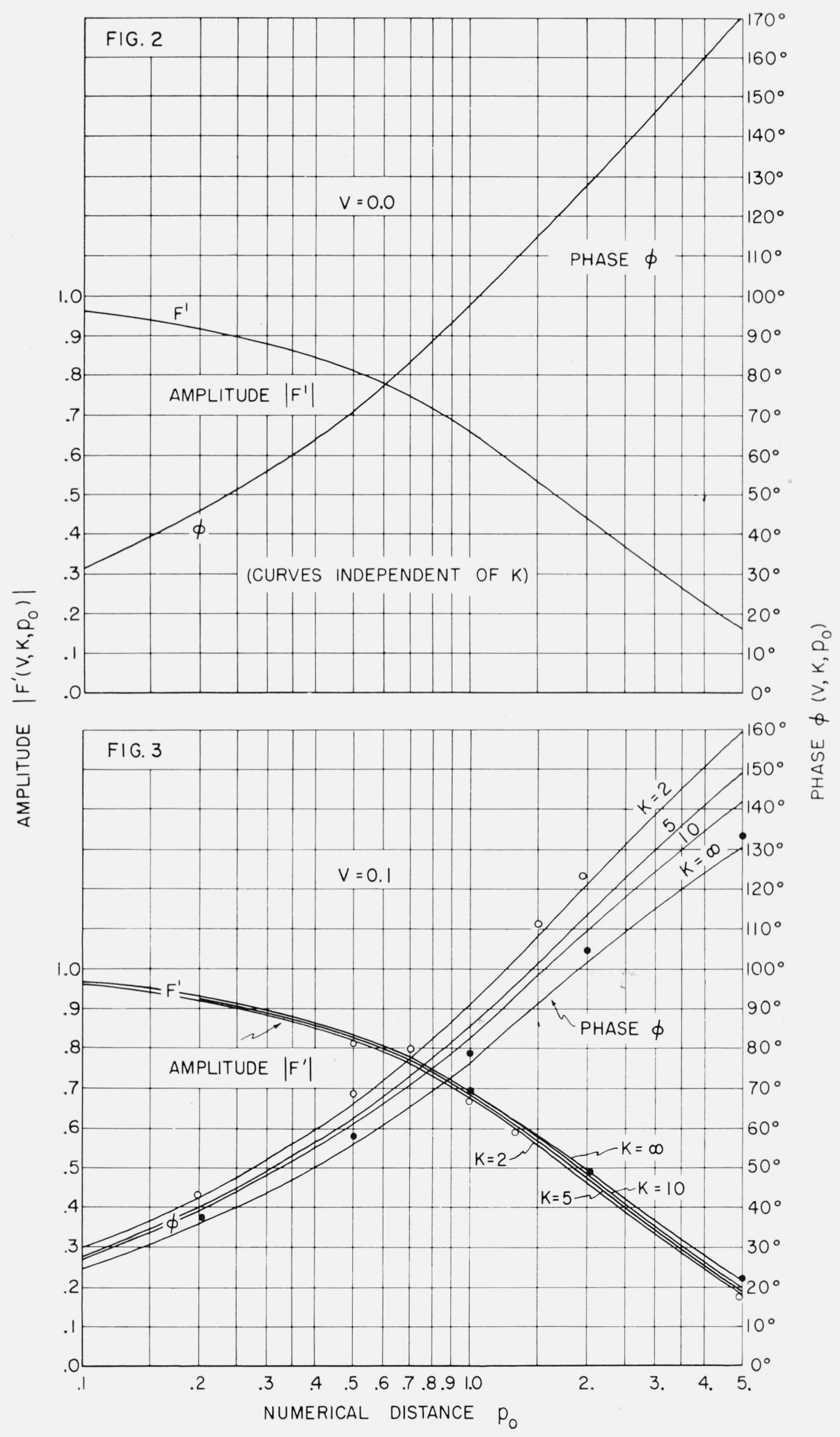

Figures 2 ANd 3. Attenuation function $\left|F^{\prime}\right| e^{i \varphi^{\prime}}$ for transmission between $A$ (or $B$ ) and $B$ (or $A$ ) over a mixed path plotted as a function of the numerical distance $p_{0}$. 


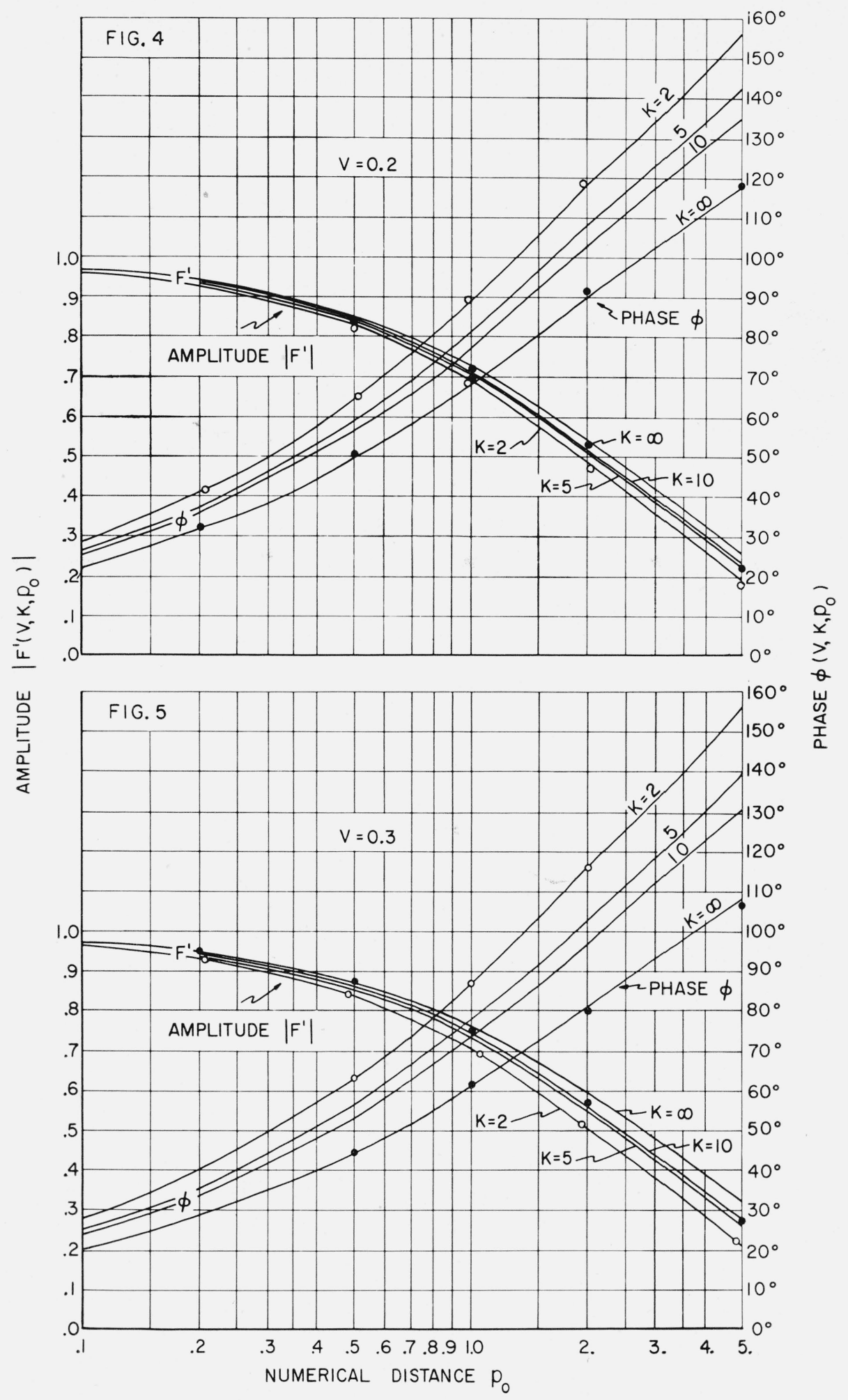

Figures 4 ANd 5. Attenuation function $\left|F^{\prime}\right| e^{i \varphi^{\prime}}$ for transmission between $A$ (or $B$ ) and $B$ (or $A$ ) over a mixed path plotted as a function of the numerical distance $p_{0}$. 


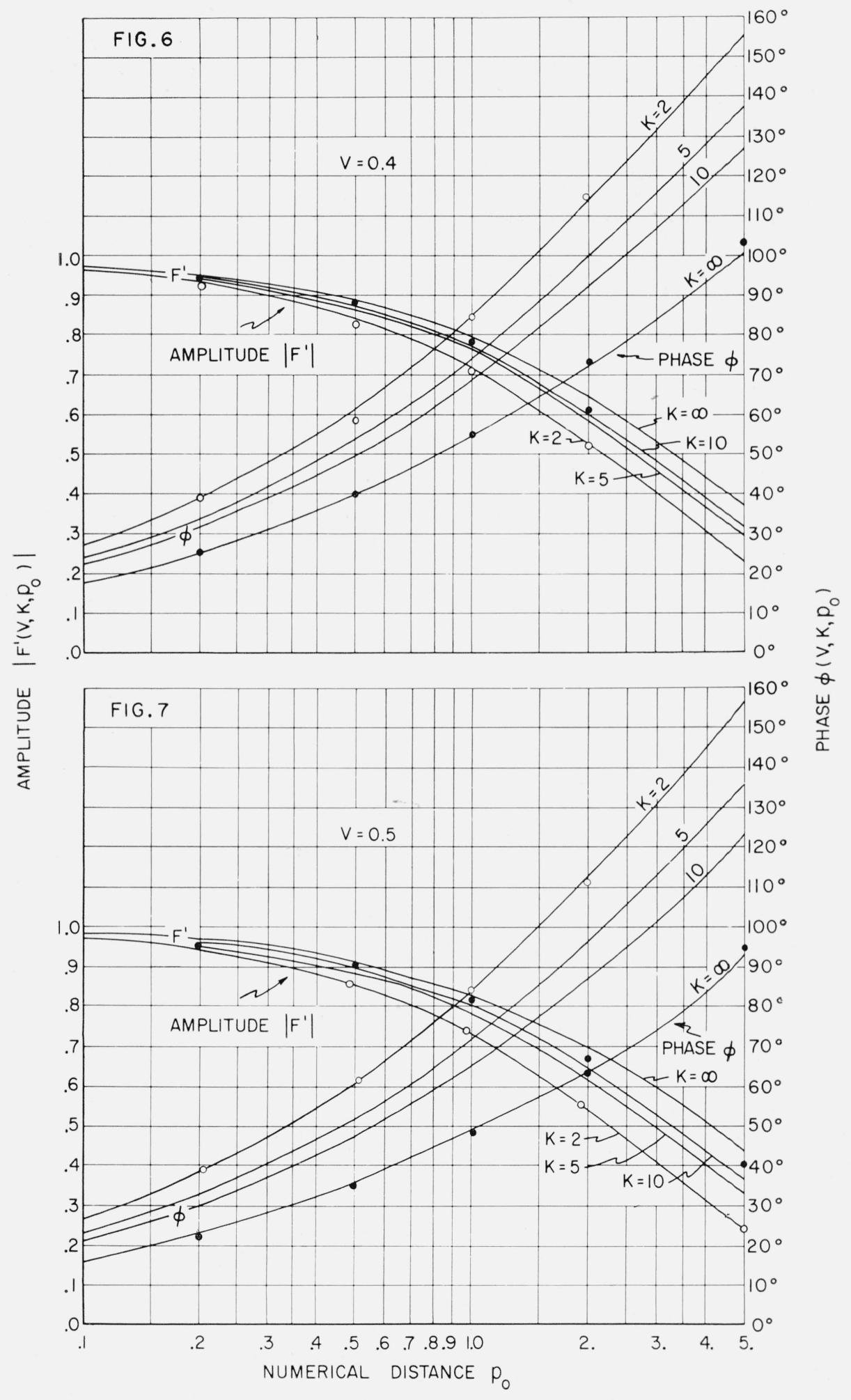

Figures 6 AND 7. Attenuation function $\left|F^{\prime}\right| e^{i \varphi^{\prime}}$ for transmission between $A$ (or $\left.B\right)$ and $B$ (or $A$ ) over a mixed path plotted as a function of the numerical distance $p_{0}$. 


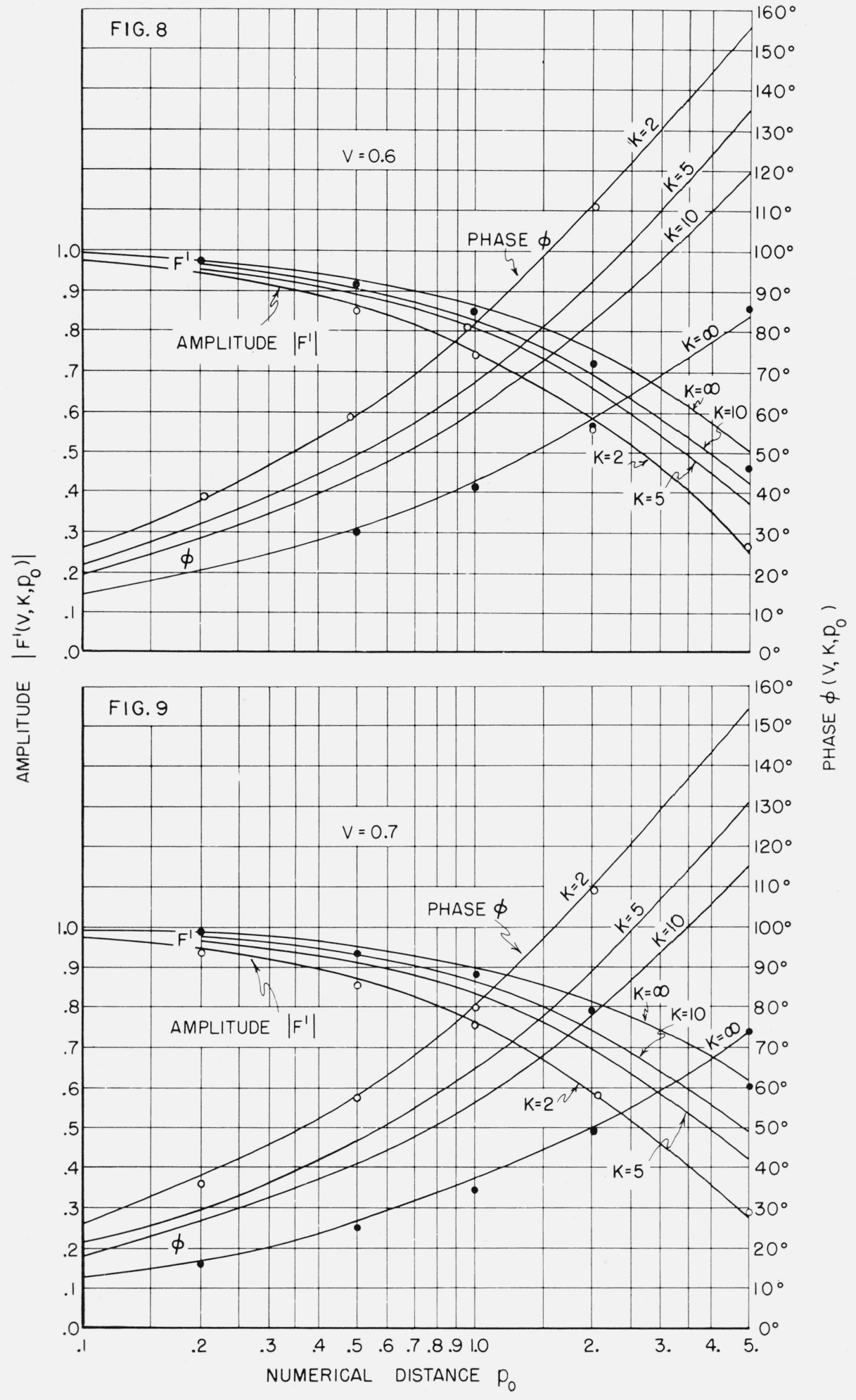

Figures 8 And 9. Attenuation function $\left|F^{\prime}\right| e^{i \varphi}$ for transmission between $A$ (or $\left.B\right)$ and $B$ (or $\left.A\right)$ over a mixed path plotted as a function of the numerical distance $p_{0}$. 


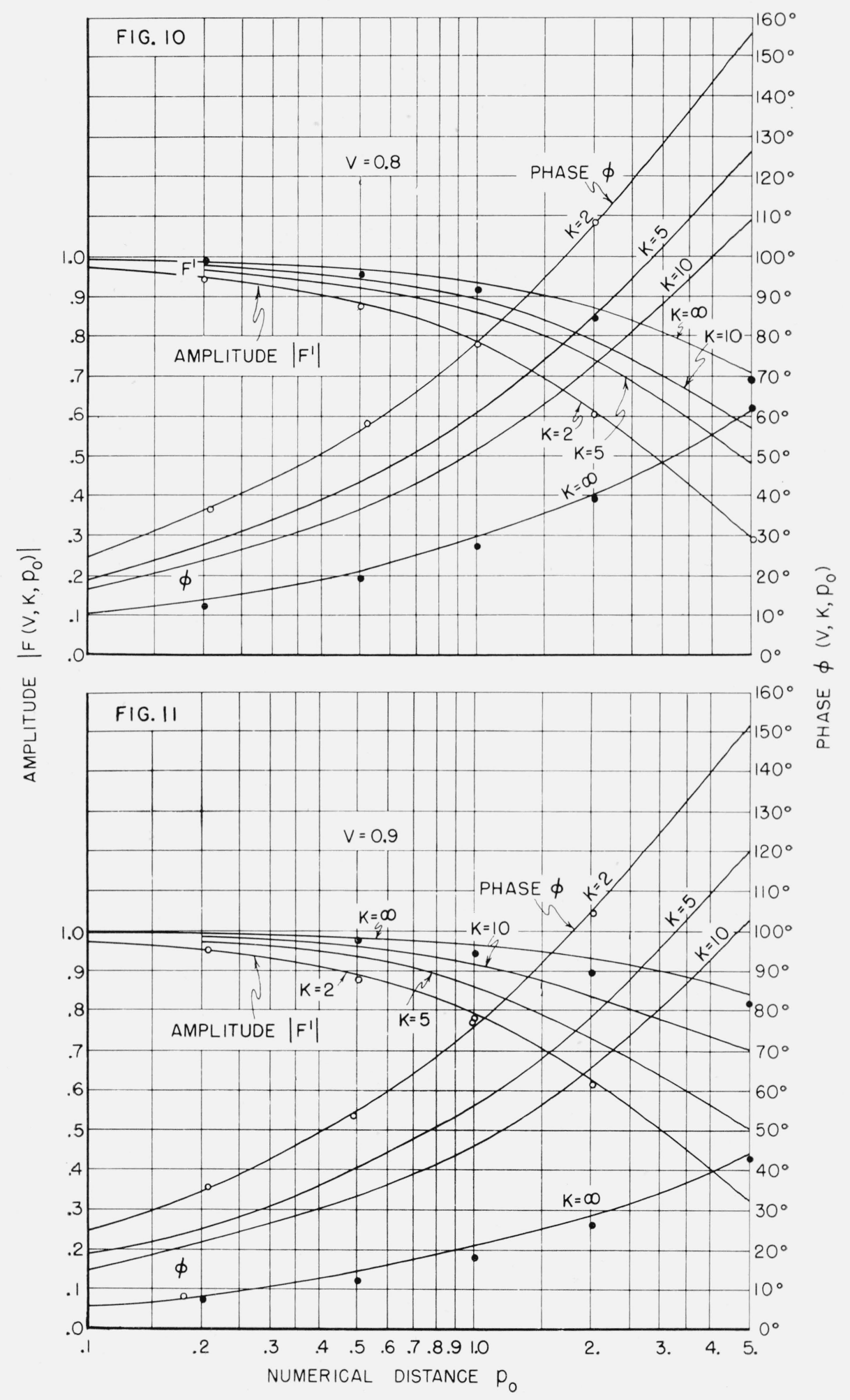

Figures 10 and 11. Attenuation function $\left|F^{\prime}\right| e^{i \varphi^{\prime}}$ for transmission between $A$ (or $B$ ) and $B$ (or $A$ ) over a mixed path plotted as a function of the numerical distance $p_{0}$. 

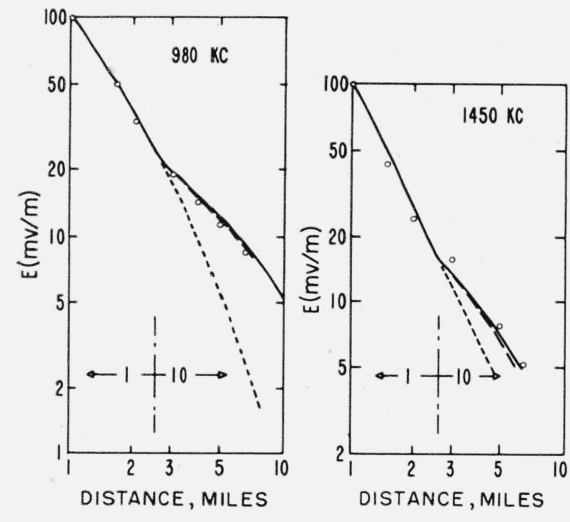
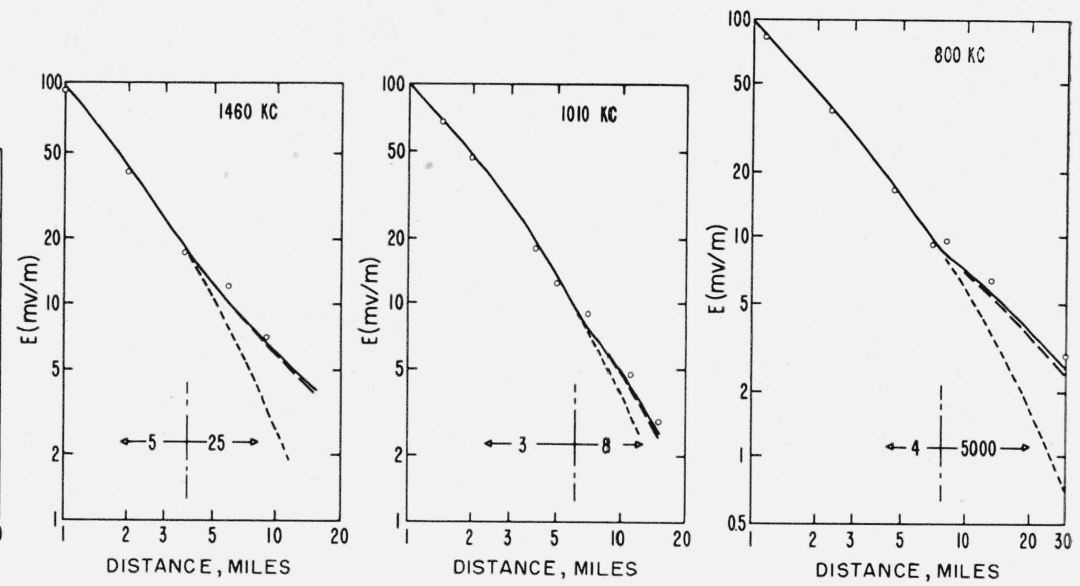

FIGURE 12. Field strength versus distance curves for various mixed paths.

The conductivity in millimhos per meter to the left and right of the boundary is shown on each curve. The experimental results were communicated to the author privately by P. A. Field, formerly of the Canadian Broadcasting Corporation. ——_, From parametric curves; — —, computed by Milling. ton method; $\bigcirc$, experimental (P. A. Field); - - ---, curve, assuming homogeneous ground throughout $(V=K=0)$.

\section{An Application}

To illustrate the application of these parametric curves, the field strength in millivolts per meter is shown plotted in figure 12 as a function of the distance $\left(=r_{0}+R_{0}\right)$ in miles for five different mixed paths. The curves are normalized so that the field strength at 1 mile is $100 \mathrm{mv} / \mathrm{m}$. Experimental values supplied by P. A. Field are also indicated. The curves calculated by the Millington method are also shown in figure 12. The conductivities on the near and far sides of the boundary are indicated on the figures and are expressed in millimhos per meter. The agreement between the computed and experimental results is quite good. For these short distances the curves calculated by the Millington method fell slightly below the curves computed from the integral formula. In view of the uncertainty in the exact physical features of the ground, the difference between the two methods of calculation hardly seems significant for these situations. It is interesting, however, to note that the Millington method in four out of five cases underestimates the recovery effect to a greater degree than the integral method. The dotted curves in figure 12 correspond to the case where the ground is homogeneous throughout, and has the conductivity of the earth on the transmitter side of the boundary. The difference between the solid and dotted curves is a measure of the recovery effect, and it is apparent that the theory indicates a gradual transition at the boundary and does not show any sudden or transient features at the boundary. It would seem from the experimental results that there is actually some type of disturbance at the boundary between the two media which is not predicted by the theory. It is believed that a more rigorous evaluation of the integral equation is necessary to describe the nature of the field near the boundary.

\section{The Refraction Effect}

Although this problem. was formulated for the mutual impedance between antennas $\mathrm{A}$ and $\mathrm{B}$ on either side of an oblique boundary, the consequent path of stationary phase did not depend on the inclination angle $\theta$. In other words, any refraction effects were neglected. It is the purpose of this section to revise the stationary phase evaluation of the integral to account for the changes of phase velocity between the two media. It is admitted that the procedure is not rigorous and is based on certain physical ideas that have their roots in geometrical optics.

With regard to figure 1, the direct ray $\mathrm{AOB}$ between the antennas would represent the path of stationary phase if refraction effects were neglected. Of course, if the boundary was at a 
right angle to $\mathrm{AOB}$ (i. e., $\theta=90^{\circ}$ ), it would be rigorously justified to assume $\mathrm{AOB}$ was the path of the stationary phase. It can be expected, however, that for the oblique boundary the path of stationary phase would be along a line APB, where P was displaced along the boundary from $\mathrm{O}$ by an amount $q$. Furthermore, it can be anticipated that $q$ would be small compared to $r_{0}$ and $R_{0}$. The location of the point $\mathrm{P}$ can be best obtained by regarding $q$ as a variable quantity in order to find when the total phase along APB is stationary or when it is a minimum. This is essentially a statement of Fermat's principle in optics. The difference between the phase along the paths $\mathrm{AOB}$ and $\mathrm{APB}$ is now considered to be due to two factors: (1) the actual increase of path length, and (2) the change of the ratios of the path lengths for the two respective media. For example, if the left-hand medium is more poorly conducting than the righthand medium (i. e., $K>1$ ), the phase velocity is relatively less to the left of the boundary, so that AP is less than AO. More explicitly, the total phase $\Phi$ over the path APB can be written

where

$$
\Phi(q)=\beta\left(\hat{r}_{0}^{\prime}+\hat{R}_{0}^{\prime}\right)+\phi^{\prime}\left(\hat{V}, K, \hat{p}_{0}\right)
$$

$$
\begin{aligned}
& \hat{r}=\left[r_{0}^{2}+q^{2}-2 r_{0} q \cos \theta\right]^{1 / 2} \\
& \hat{R}_{0}=\left[R_{0}^{2}+q^{2}+2 R_{0} q \cos \theta\right]^{1 / 2} \\
& \hat{V}=\frac{\hat{R}_{0}}{\hat{r}_{0}+\hat{R}_{0}} \text { and } \hat{p}_{0}=-\frac{i \beta\left(\hat{r}+\hat{R}_{0}\right)}{2}\left(\frac{\eta}{120 \pi}\right) .
\end{aligned}
$$

The function $\phi\left(\hat{V}, K, \hat{p}_{0}\right)$ in the above expression is taken to be of the same form as the function $\phi\left(V, K, p_{0}\right)$ previously computed. The general scheme is then to vary $q$ to find where $\Phi(q)$ is stationary. For the present purpose, however, it is convenient to make some further approximations utilizing the fact that $q$ is small. The phase function then becomes

$$
\Phi(q) \simeq \beta\left(r_{0}+R_{0}\right)\left[1+\frac{\sin ^{2} \theta}{2}\left(\frac{q}{d}\right)^{2} \frac{1}{V(1-V)}\right]+\phi^{\prime}\left(\hat{V}, K, \hat{p}_{0}\right),
$$

where

$$
\hat{V} \simeq V+\frac{q}{d} \cos \theta, \quad d=r_{0}+R_{0}
$$

and

$$
\hat{p}_{0} \simeq p_{0}\left[1+\frac{\sin ^{2} \theta}{2}\left(\frac{q}{d}\right)^{2} \frac{1}{V(1-V)}\right] \simeq p_{0}
$$

The terms containing higher powers in $(q / d)$ have been neglected and, in fact, in the expression for $p_{0}$, the term containing $(q / d)^{2}$ can be also dropped, since $\phi^{\prime}\left(V, K, p_{0}\right)$ is a relatively slowly varying function of $p_{0}$. It is now convenient to express $\phi^{\prime}(V)$ as a 'Taylor expansion, as follows:

$$
\phi^{\prime}(\hat{V})=\phi^{\prime}(V)+\frac{q \cos \theta}{r_{0}+R_{0}}\left[\frac{d \phi^{\prime}(\hat{V})}{d \hat{V}}\right]_{\hat{v}=v}+\left[\frac{q \cos \theta}{r_{0}+R_{0}}\right]^{2} \frac{1}{2}\left[\frac{d^{2} \phi^{\prime}(V)}{d \hat{V}^{2}}\right]_{\hat{v}=v}
$$

Because $q$ is small compared to $r_{0}+R_{0}$, only the first term or two of the expansion are significant. The phase function now becomes

$$
\Phi(q) \simeq \beta\left(r_{0}+R_{0}\right)+\phi^{\prime}\left(V, K, p_{0}\right)-\frac{q \cos \theta}{r_{0}+R_{0}} \delta(V)+\frac{\sin ^{2} \theta}{V(1-V)} \pi \frac{d}{\lambda}\left(\frac{q}{d}\right)^{2},
$$

where $\delta(V)=-d \phi(V) / d V$ is essentially a positive quantity. The right-hand side of the equation is a quadratic in $q$ and has a minimum or stationary value when

$$
\frac{q}{d}=\frac{q_{0}}{d}=\frac{\cos \theta \delta(V) V(1-V) \lambda}{2 \sin ^{2} \theta \pi\left(r_{0}+R_{0}\right)}
$$


This value of $q$, namely, $q_{0}$, which makes $\Phi(q)$ stationary, is then a measure of the deviation of the direction of the phase of the ground wave as it crosses the boundary. The appropriate values of $\delta(V)$ can be obtained directly from the parametric curves of $\phi^{\prime}\left(p_{0}, V, K\right)$. Actually, it is a little more meaningful to consider the angles $\delta$ and $\delta_{1}$, which are the angles OBP and OAP in figure 1. These can be called bearing errors, as they are a measure of the change of direction of the phase front relative to a direct unimpeded wave between $\mathrm{A}$ and $\mathrm{B}$. In terms of $q_{0}$, the bearing errors, expressed in degrees, are given by

$$
\delta \simeq \frac{q_{0}}{r_{0}+R_{0}} \frac{1}{(1-V)} \sin \theta \frac{180}{\pi}
$$

and

$$
\delta_{1} \simeq \frac{q_{0}}{r_{0}+R_{0}} \frac{1}{V} \sin \theta \frac{180}{\pi} .
$$

Employing typical values of the parameters and ietting $K=\infty$, values of $\delta$ and $\delta_{1}$ were com-

\begin{tabular}{|c|c|c|c|c|c|c|}
\hline \multirow{2}{*}{$V$} & \multirow{2}{*}{$\frac{\epsilon_{0} \omega}{\sigma}$} & \multirow{2}{*}{$\frac{r_{0}+R_{0}}{\lambda}$} & \multicolumn{2}{|c|}{$\theta=45^{\circ}$} & \multicolumn{2}{|c|}{$\theta=60^{\circ}$} \\
\hline & & & $\delta$ & $\delta_{1}$ & $\delta$ & $\delta_{1}$ \\
\hline 0.2 & 0.01 & 50 & 0.06 & 0.24 & 0.03 & 0.14 \\
\hline 2 & .01 & 100 & 03 & .14 & .02 & .08 \\
\hline 2 & 001 & 50 & .02 & .08 & 01 & \\
\hline 2 & 001 & 100 & .01 & .06 & 01 & \\
\hline .5 & 01 & 50 & .10 & .10 & .06 & .06 \\
\hline .5 & 01 & 100 & .06 & .06 & .03 & .03 \\
\hline .5 & .001 & 50 & .03 & .03 & .02 & .02 \\
\hline .5 & 001 & 100 & .02 & .02 & 01 & .01 \\
\hline 8 & 01 & 50 & .26 & .06 & .17 & .04 \\
\hline 8 & 01 & 100 & .16 & .04 & .09 & .02 \\
\hline .8 & .001 & 50 & .08 & .02 & .05 & .01 \\
\hline 8 & 001 & 100 & .06 & .02 & .04 & .01 \\
\hline
\end{tabular}
puted and are:

It might seem surprising at first glance that these values are so small. This is as should be expected, however, because the absolute phase velocities of the ground wave over the two media differ only by a fraction of one percent [10]. It is noted that the bearing errors are largest when the antenna is near the boundary, and where the separation between antennas $\mathrm{A}$ and $\mathrm{B}$ is 50 wavelengths or less. Of course, as the obliqueness of the boundary becomes more noticeable (i. e., $\theta<45^{\circ}$ ) the calculated values of $\delta$ and $\delta_{1}$ would be larger. One should be cautious, however, in attempting to apply this method to the case where the boundary makes a small angle with the line $\mathrm{AB}$ between the antennas. In such an instance the approximation used in evaluating the basic integral by a stationary phase principle becomes invalid.

\section{Conclusion}

The results obtained in this paper provide a theoretical check of the Millington semiempirical method of calculating the amplitude and phase of a wave crossing a boundary separating two media. The parametric curves presented here should be convenient for making predictions for propagation along a two-media path at low and medium frequencies. It is also indicated that the refraction effects for a wave crossing the boundary obliquely are very small.

I record my thanks to Loris Perry, who carried out most of the computations. 


\section{References}

[1] K. Suda, Field-strength calculations-new method for mixed paths, Wireless Engineer 31, 249 (1954).

[2] H. L. Kirke, Calculations of ground wave field strength over a composite land path, Proc. Inst. Radio Engrs. 37, 489 (1949).

[3] G. Millington, Ground wave propagation over an inhomogeneous smooth earth, part 1, Proc. Inst. Elec. Engrs. 96, 53 (1949).

[4] P. C. Clemmow, Radio propagation over a flat earth across a boundary separating two different media, Trans. Roy. Soc. (London) 246, 1 (1953).

[5] H. Bremmer, The extension of Sommerfeld's formula for the propagation of radio waves over a flat earth to different conductivities of the soil, Physica 20, 441 (1954).

[6] E. Feinberg, Propagation of radio waves over an actual surface, Izdatel'stbo Akademii Nauk S. S. S. R. (Moscow), 109-131 (1944).

[7] S. Ballantine, Reciprocity in electromagnetic mechanical, acoustical, and interconnected systems, Proc. Inst. Radio Engrs. 17, 929 (1929).

[8] G. D. Monteath, Application of the compensation theorem to certain radiation and propagation problems, Monograph No. 3, Proc. Inst. Elec. Engrs. 98, 1 (1951).

[9] K. A. Norton, Calculation of the ground wave field intensity, Proc. Inst. Radio Engrs. 29, 623 (1941).

[10] J. R. Wait and L. L. Campbell, Transmission curves for ground wave propagation at low radio frequencies, Report R. 1, Defence Research Telecommunications Establishment, Ottawa, Canada (1952). (Extensive amplitude and phase data are given in this report for frequencies from 15 to $500 \mathrm{ke}$ for a wide range of conductivities and dielectric constants.)

Boulder, December 7, 1955. 